posed in accepting a family history of Marfan syndrome it is possible this figure will increase. Failure of consultant diagnosis: It was not possible to exclude a familial cardiac disease in $33 \%$ of consultants referred to this clinic, usually because of young age. Failure of diagnosis poses problems in counselling families and $80 \%$ of all individuals referred continue to be followed up. Decision to not be screened: $14 \%$ of asymptomatic family member declined further examination and investigations after genetic counselling. This suggests that individual expectations of screening may be unrealistic. Ethical Issues: In some cases family members who had been not referred to this clinic were identified as obligatory carriers or being affected. This poses ethical dilemmas in decisions to disclose or undisclose this information to those involved. Conclusions: Requests for screening are likely to continue to increase, placing a considerable burden upon existing resources. The diagnostic capabilities of even a specialised clinic are limited and it is likely that many individuals will be followed up uncertain of their diagnosis. Increasing ethical issues are likely to be faced. Local policies for meeting these problems should be addressed now.

\section{General Paediatric Cardiology}

\section{2}

Myocarditis in children - correlation of clinical and immunohistological diagnosis

Ziölkouska L, Kubicka K, Woznieuricz B, Kowalik G, Kawalec W, Mrouvec L, Brzezinska-Rajszys $G$

Dept. of Paediatric Cardiolo., The Children's Memorial Health Institute, Warsau, Poland

Myocarditis (MYO) in children can be symptomatic (congestive heart failure, arrhythmia, chest pain) or totally asymptomatic. Myocarditis can be cause of sudden death, persistent inflammatory process with/without clinical symptoms, dilated cardiomyopathy (DCM) or arrhythmogenic right ventricular dysplasia (ARVD). The aim of study was: 1 . to compare the results of endomyocardial biopsy (ENB) in children with clinical diagnosis of MYO, DCM and ventricular arrhythmias. 2. to evaluate the diagnostic value of EMB in MYO in children. There were $55 \mathrm{pts}$ ( 32 girls, 23 boys, age $1-17,4 y r s(x=8,6 y r s)$ divided into 3 groups. In all pts following tests were done: antiviral Coxackie B 2,3,4, Parainfluenza 1,2,3 and antimyosin antibodies, routine and $24-\mathrm{h}$ Holter ECG, echocardiographic evaluation of left ventricle diameter and function, chest X-rays, MRI and EMB. In EMB 3 specimens from RV were evaluated:

I specimen - lymphocytic index (IL) using Dallas criteria, Il specimen - immunohistological typing of lymphocyte $T$ and $B$ using specific monoclonal antibodies (CD3, CD4, CD8, CD22, CD31, $\mathrm{CD} 68$ ) and activation of adhesion molecules (Intercellular Adhesion Molecule = ICAM, Vascular Adhesion Molecule = VCAM, Endothelial Adhesion Molecule = ELAM), III - specimen ultrastructural evaluation of degenerative hypertrophy and ARVD.

Conclusion: 1. Histological features of myocarditis were found in $71 \%$ pts with clinical diagnosis of MYO, $67 \%$ of DCM and $38 \%$ of ventricular arrhythmias. 2. EMB should be performed in children not only in myocarditis but also in other myocardial diseases false positively diagnosed as DCM and ventricular arrhythmias of unknown etiologv. 3. In children the sequeles of myocarditis can be DCM and ARVD.

\section{3}

High molecular weight heparin for protein-losing enteropathy (PLE) after Fontan operation

Hain U, Saner U, Hauser M, Brodherr S, Hess J, Heimisch W, Randak $C^{\star}$, Malcic I**

Deutsches Herzzentrum München (DHM), *Haunersches Kinderspital Mïnchen $D, \star \star$ University Hospital Rebro Zagreb, Croatia

Between 07/1974 and 07/1994, 175 pts underwent modified Fontan op. (FO) at a mean age of 8.5 y at DHM. In 24 pts (14\%), PLE was diagnosed on the basis of chronically low total serum protein (TP) levels $(<5 \mathrm{~g} / \mathrm{dl})$ with edema, effusions, ascites, and diarrhoea and elevated mean RAP (15 mmHg). 11 pts with PLE died. In 5 pts, PLE resolved under medical or surgical treatment of pulmonary branch stenosis. In 8 pts, high molecular weight heparin (HMWH) therapy with $5000-6000 \mathrm{U} / \mathrm{m} 2$ s.c.once a day was begun after a mean period of PLE of 5 y (1.5-9 y). Reoperation for relief of obstruction in the FO circulation in $5 \mathrm{pts}$ did not improve PLE. Death of $1 \mathrm{pt}$ after $7 \mathrm{~d}$ of HMWH seemed unrelated to therapy.

During follow-up of the remaining 7 pts (3-6 mo) all PLE symptoms decreased by $2 / 3$ under HMWH. After a steep increase of TP within the first $7 \mathrm{~d}$, a levelling off over the next $3 \mathrm{w}$ occurred. In 4 responders the positive effect persisted, whereas the TP levels remained $<5 \mathrm{~g} / \mathrm{dl}$ in 3 pts. Transient decrease in TP in 3 responders was due to acute gastroenteritis. The changes in TP levels correlated well with fecal $\alpha_{1}$-antitrypsin values. Except for local bleeding at puncture site, no further complications were observed under strict control of clinical and laboratory parameters. Response to heparin therapy can be expected after 1 mo and scems to be independent of PLE duration. This preliminary experience does not allow judgement on therapy duration and minimal effective heparin dose.

\section{4}

Stress-testing in children with TGA after arterial switch op: exercise capacity, ventricular function and myocardial perfusion

Hauser $M$, Kïln $A$, Braun S, M. Schwaiger $M$, Hess J

Deutsches Herzzentrum München, Nuklearmedizinische Klinik der TUMïnchen, FDR

22 children (age $12.3 \pm 2.2$ years) with transposition of the great arteries (TGA) were investigated in mean $11.2( \pm 2.9)$ years after arterial switch operation. Spiroergometry (treadmill, Bruce protocol) was performed in all patients; there was no significant difference in cardiopulmonary exercise capacity (anacrobic threshold, maximal oxygen uptake) between patients and normal matched volunteers. On stress echocardiography there was a normal exercise induced increase of ventricular function in all patients; two of them had dyskinetic areas within the left ventricular myocardium. There was a significant increase of glycogenphosphorylase activity as a marker for myocardial ischaemia after exercise in five patients; four of them had adenosin induced perfusion defects in positron emission tomography. Coronary flow reserve (CFR) was significantly reduced on PET scanning in all patients compared to normal healthy volunteers.

Conclusion: Children after arterial switch operation have a normal cardiopulmonary exercise capacity; nevertheless exercise induced ischaemia is apparent in some patients, whilst being clinical asymptomatic. Coronary insufficiency, reduced CFR and exercise induced perfusion defects may cause major problems in adulthood. 